\title{
A Survey and Analysis of Cloud Social Media Environment with QoS Aware System
}

\author{
Sumanth $^{1}$, S. Sailaja ${ }^{2}$ \\ 1, 2Computer Science Engineering, Rise Group of Institutions, Ongole, India
}

\begin{abstract}
Popularity of mobile devices network technologies increases day by day. Mobile devices always maintain network connectivity by different network providers. So if user moves around then they can access cloud services without any disadvantages. In current model when user moves from one geographical area to another he will keep accessing services from previous cloud over a long distance. It results in more congestion on network. This will degrades the QoS and QoE of services in cloud. There is the need of different approach which maintains resources by improving QoS and QoE of mobile services. This framework tells that the services run on public cloud are able to populate to other cloud in different location. This paper proves that if we add resource pool for every cloud then it is responsible for removing ambiguity which occurs at the time of migrating services. It also find out how the number of clients can influence the decision making at service delivery layer.
\end{abstract}

Keywords: cloud computing, QoS, QoE, service population

\section{Introduction}

Cloud computing becomes popular now a days because of its simple nature. It offers various computing and storage services over a internet. Cloud service providers rent data centers hardware and software to deliver storage and computing services through the internet. Internet users can access services from cloud. Instead of their own devices cloud users can store their data on cloud. They can run their applications on cloud platform without full installation of software. Cloud service providers provide various cloud services and resources as user requirement and they charged them accordingly. Cloud computing increases its popularity because of its simple nature. Amazon EC2 and Applels iCloud are very popular cloud based products [2]. Those vendors create their own cloud services and offer them to client for business and individual uses. They create cloud services as requirements come from market and each have different than others. Mobile computing also becomes more popular due to smartphones and tablet pcs. Laptops and desktops are cannot be easily operate due to its size and form. So it increases the demand of less weighted mobile devices than laptops and desktops. But these devices cannot have some hardware resources which is used to perform some critical task. At that situation there is the need to access those devices remotely through network for storage and processing. This feature is provided by cloud computing. It provides center based resources and those devices require decenter based pool of resources. It creates traffic congestion problem on internet due to user mobility and high bandwidth services. It affects QoS and QoE factors in mobile services. This paper consists of framework which overcomes the problem by service populating technique.

\section{Literature Survey}

A previous project invents a reshaping of the physical footprint of virtual machines within a cloud [3]. It invents a concept towards the lower operational costs for cloud providers and improvement of hosted application performance by taking into account affinities and conflicts between replaced virtual machines. It is achieve by mapping virtual machine footprints. After comparing if similarities found in memory footprint the virtual machines are migrated to the same memory location and content based memory sharing also deployed to get consolidation[ 4]-[6].The basic thing is to build control system for cloud which perform footprint reshaping to achieve higher level objectives like low power consumption, high reliability and better performance. It then reduces the cost for cloud providers and creates low cost cloud services for user.

Media Edge Cloud (MEC) architecture improves the performance of cloud technology. This architecture also improves QoS and QoE for multimedia applications. To achieve that cloudlets of servers running at edge of bigger cloud. So it handles the request closer to the cloud thus it reduces the latency. If requests needs further processing then requests are sent to the inner cloud due to that the cloudlets are reserved for QoS based multimedia applications [7]. Using that concept the physical machines closer to the clouds outer boundary will used to handle QoS sensitive services. As these machines located on outer boundary of cloud the data has to travel less distance within the cloud before sending to the client. It improves QoE for client and reduces network congestion of cloud. All these researches aims only to improve the cloud performance, no one can think about the user mobility. Providing media services to mobile clients becomes popular in future. As per that concept mobility and multimedia contents becomes more popular and high bandwidth data streams will have to travel more distance and reach moving target can create a problem in future. Cloud providers may need to create more clouds to handle the load and reduces the congestion. In cloud computing client get services by contacting a physical resources directly and then ask about the service. Clients need to connect to the cloud then they can accesses the services from the cloud. But in this approach client need to know the name of the physical resource which offers the services to the client, so it creates the problem of redundancy. Some organizations solve this problem by running multiple servers and by using DNS, for load, balancing and fail over [7]. This approach needs more cost which is not affordable for small entities which offers a service at lower layer. The ability for clients to request 


\section{International Journal of Science and Research (IJSR) \\ ISSN (Online): 2319-7064}

Index Copernicus Value (2013): 6.14 | Impact Factor (2014): 5.611

services directly from the network instead of asking for physical resources that offers those services[1]. It opens a doors for future development. Client request a service ID and network infrastructure which is used to find whether the actual service is running and then connect it to the client. This approach is able to running a service in multiple locations and directly client requests to the appropriate instance depending on their location and network status.

\section{Programmer's Design}

\subsection{System Model}

QoS aware service delivery model is necessary to deliver the services. The network infrastructure is used to decide the network status between the client and service. Service providers provide services with best QoS and QoE parameters to their clients. In this model client of cloud services will remain connect to the same cloud without thinking about its physical location and network status. If the network condition satisfactory and there is no redundant path the service will be out of reach of network. So providers not able to reach their SLA standards and clients not getting the best QoE at all time. Another thing is that the cloud from any location has connected to the same cloud to get services without thinking about the distance of cloud from itself. It results in creating more processing load on cloud which degrades the QoS of services. It is not possible for cloud providers to build a multiple clouds to provide services to the different geographical areas. So there is the need of new technique for service delivery which provides various services to clients with proper QoS and QoE parameter, it is also provide better cloud management to the providers, it also reduces the network congestion. In this service delivery model we will have clients who request the services and their requests will be directed to physical location at which the service is running by fulfilling QoS and QoE parameters. In the case of mobility it is difficult to direct client to a specific instance of service. We can connect client to the service instance based on their present location and network conditions, but if client move to another location with different network area then it is difficult to get this. If the user moves far away from the cloud then it creates congestion on network so it impacts on the QoS of all services on the network. To solve this problem we could connect to the client at different instance of service each time the QoS parameter degrades, at that time not expected to create multiple clouds by cloud providers. Single cloud providers may not own multiple clouds at different physical location so it is possible that many cloud providers have their cloud far apart or down to regional scale within a country. So we able to address the issue of service population across the different boundaries of cloud providers. It introduces a concept where service providers will register their services globally and not bound to specific cloud providers. Services which are globally registered and not bound with specific cloud providers will free to populate or migrate to different cloud depending on QoS and source of service request parameters. This will only possible when cloud providers open their boundaries, so services can move in and out of their cloud. It will change the model of service providers. Service providers will register their services with service level agreement which defines the expected QoS parameters.
Cloud providers provide services with best QoS so that it will populate their service and it gives income for them. It is not possible to any big cloud to take all the services due to the network congestion problem. So the services may populate from bigger cloud to smaller cloud to maintain network congestion free and minimize the distance of itself from client. After populating services from one cloud to another the receiving cloud can also reject the populating service, if it is already under the heavy load. This population of service process is completely transparent to the user. To achieve all those things there is the need of new service delivery framework and it should be QoS aware and support service population. At the time of migration of any service from one cloud there may be the chance that another user is accessing the same service so after migration of service from current cloud leads to starvation of second client. So to solve this problem we add separate resource pool to each cloud which is used to keep references or object of all populated and nonpopulated services. Another client can access the populating service without any interruption.

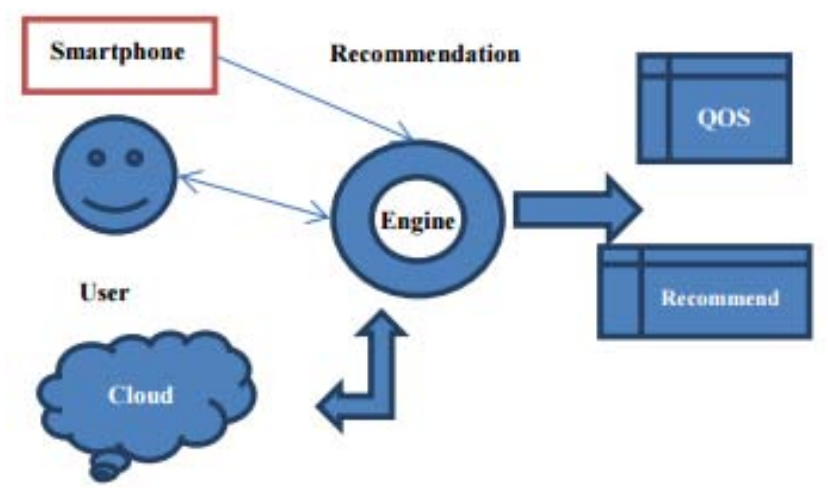

Figure 1: System Architecture

Above figure shows a system architecture smartphones and users are the clients who accessed the services of cloud. Those are mobile clients so if they move from one location to another then there is the need to populate the services to another location. So the engine gives the recommendations depending on the QoS parameters. The another cloud decides whether to +

\section{Mathematical Model}

We apply set theory to our project. The aim of project is to perform service migration to improve QoS \& QoE of a service. Let there are three sets one is Cloud, one is service population and one is QoS . Cloud is union of QoS and population.

So we will consider some QoS requirements and some populating services from set population which are corresponding to each other.

We will merge these sets by intersecting them as shown in following fig. Hence we get the solution to our project, by populating some services from one cloud to another with satisfying QoS parameter we can manage the traffic \& removes the ambiguity.

\section{Volume 4 Issue 12, December 2015}


International Journal of Science and Research (IJSR)

ISSN (Online): 2319-7064

Index Copernicus Value (2013): 6.14 | Impact Factor (2014): 5.611

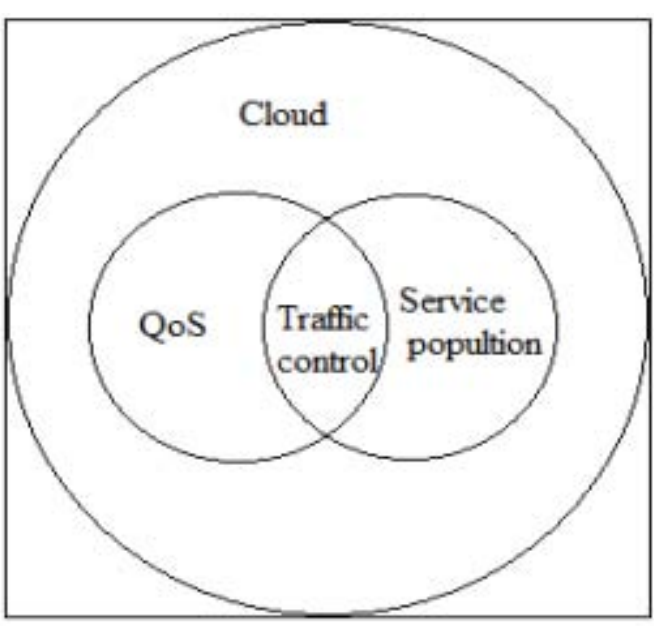

Figure 2: Venn diagram

The service populating model needs a concept of an open cloud. The existing closed cloud only runs services controlled by its owner. Open cloud allows services from third party providers to populate it. The open cloud is like resource pool so anyone can use these resources to run their services and anyone can provide such resource pool and accept services from other providers to run on it. So the new framework comes in model. This proposed Framework consists of six layers.

\subsection{Service management layer}

It is used to check how services are registered in a cloud. Billing information between resources and service providers is processed. It is considered as part of application layer in OSI because it defines the applications and how they use resources. When service providers want to publish service they have to define security QoS parameters. It is the requirement to run the service. So to do that each service must have a list of parameters this must agree with parameters defined by cloud. It is also used to migrate the service to find proper clouds that can accept the service. At that time if service needs an extra resource, it can be given as per that service providers will billed.

\subsection{Service Subscription Layer (SSL)}

It is used to perform the subscription of clients to the service the information which handle the subscription, like user IDs, list of services subscribe by individual client. Subscriber is clients who request a service and he will be charged for subscription. It is used to keep track of number of from which location accessing a service.

\subsection{Service Delivery Layer (SDL)}

It delivers services to specific clients. It is responsible for publishing a service from one cloud to another cloud. Finding the appropriate cloud as per the necessary requirements is done and then service is populating to this cloud.

\subsection{Service Migration Layer (SMiL)}

The migration of services between clouds is the responsibility of SMiL. To populate a service we have to first sure that the target cloud can accept the service. So the decision of whether to move or not to move a service is done at SDL. Using that decision SMiL instruct the cloud about which resources need to be allocated.

\subsection{Service connection layer}

It handles the client mobility issue and also checks the connection between client and services. F. Service network abstraction layer It provides the abstraction property to simplify the migration process. It acts as interface between service delivery framework and new technology

\section{Algorithm}

1) Create and start node.

2) Start QoS manager which checks the QoS of various services.

3) Then next step is user authentication which is used to authenticate the users.

4) Authenticated user is connected to the service. 5. Suppose user access video, video streaming is going on.

5) At the time of streaming system tracks the QoS with the help of QoS manager.

6) QoS manager gives some recommendations those are track by system.

7) On the basis of recommendation system takes migration decision.

8) Service is migrated to another cloud or keeps as it is.

9) If service is migrated to another cloud system will again check the QoS.

\section{Conclusion}

This paper gives the solution on challenges presented by user mobility. Previous service delivery model is inefficient to provide future requirements of mobile user. The cloud technology with proposed model can bring the solution to proper management of network resources. This paper also introduced a technique which reduces the congestion on network, which is generated by streaming video and audio.

\section{References}

[1] Fragkiskos Sardis, Glenford Mapp, Jonathan Loo “On the Investigation of Cloud Based Mobile Media Environment with Service Populating QoS Aware Mechanisms" IEEE trasaction on multimedia, vol.15.

[2] Apple,2012.iCloudFeb15,2012.[Online] Available:http://www.apple.com/icloud.

[3] J.Sonnek and A.Chandra, Virtualputty :Reshaping the physical footprint of virtual machines, in Proc .Workshop on Hot Topics in Cloud Computing (HotCloud09), San Diego, CA, USA, Jun. 2009.

[4] J. Sonnek, J. Greensky, R. Reutiman, and A. Chandra, Starling: Minimizing communication overhead in virtualized computing platforms using decentralized Afnity-aware migration, in Proc. 39th Int. Conf.on Parallel Processing (ICPP10), San Diego, CA,USA, Sep. 2010.

[5] C. A. Waldspurger, Memory resource management in VMWare ESX server, in Proc. OSDI, 2002.

[6] T.Wood, G. Tarasuk-Levin, P. Shenoy, P. Desnoyers, E. Cecchet, and M. Corner, Memory buddies:Exploiting

\section{Volume 4 Issue 12, December 2015}




\section{International Journal of Science and Research (IJSR) \\ ISSN (Online): 2319-7064}

Index Copernicus Value (2013): 6.14 | Impact Factor (2014): 5.611

page sharing for smart colocation in virtualized data centers, in 3Proc. 5th ACM Int. Conf. Virtual Execution Environments, 2009.

[7] W. Zhu, C. Luo, J. Wang, and S. Li, Multimedia cloud computing, IEEE Signal Process. Mag., vol.28, no. 3, pp. 5969, May 2011.

[8] J. Postel and J. Reynolds, ISI, RFC 948, A Standard for the Transmission of IP Datagrams Over IEEE 802 Networks, IETF, 1988.

[9] H.Inamura, G Montengero, R Ludwig, A.Gurtov, and F.Khazov, RFC 3481, TCP over Second (2.5 G) and Third (3 G) Generation Wireless Networks, IETF, 2003.

[10]Amazon, 2012, EC2, Feb. 28, 2012. [Online]. Available:http://aws. ama- zon.com/ec2/. Microsoft, 2011CloudComputing, Feb.28, 2012. [Online].Available: http://www.microsoft.com/enus/cloud/default.aspx?fbid

[11]D.Gupta,S.Lee M.Vrable, S.Savage, A.C.Snoeren, G.Varghese, G.M.Voelker,andA.Vahdat ,Dierenceengine: Harnessing memory redundancy in virtual machines, in Proc. OSDI, 2008.

[12]T. Brisko, RFC 1794, DNS Support for Load Balancing,IETF, 1995.

[13]D. N. Thakker, Prefetching and clustering techniques for network based storage, Ph.D. dissertation,Sch. Eng. Inf. Sci., Middlesex Univ., London, U.K.,2010.

[14] Microsoft 2011, cloud computing, Feb.28;2012. [online].Available:http://www.mocrosoft,com/enus/clou d/default.aspx?fbid=uzSXFYwh_d4.

[15]ETSI, 2011, Mobile Technologies GSM, Feb.15,2012.[online].Available:http://www.etsi.org/web site/Technol ogies/gsm.aspx. 